\title{
Krebsbehandlung bei Adoleszenten - administrative, klinische und wissenschaftliche Implikationen
}

\section{Adolescents with Cancer - Administrative, Clinical and Scientific Implications}

U. Göbel, U. Creutzig, T. Klingebiel, P. Kaatsch, T. Niehues, D. Körholz

Bibliografie DOI http://dx.doi.org/ $10.1055 / \mathrm{s}-0031-1291235$ Klin Padiatr 2011; 223: 311-314 (c) Georg Thieme Verlag KG Stuttgart · New York ISSN 0300-8630

Korrespondenzadresse Prof. Dr. Ulrich Göbel ESPED-Geschäftsstelle Koordinierungszentrum für Klinische Studien Universitätsklinikum Heinrich-heine-Universität Düsseldorf Moorenstraße 5 40225 Düsseldorf goebelu@arcor.de

\section{Gesetzliche Vorgaben und administrative Änderungen $\nabla$}

In den letzten 10 Jahren hat die Medizin in allen Bereichen gewaltige Fortschritte gemacht, was nicht ohne Rückwirkungen auf die Struktur und Arbeitsweise bleiben kann. Ausgehend von einer Initiative des Europarates [13] werden unter dem gemeinsamen Dach von Bundesärztekammer (BÄK), Kassenärztlicher Bundesvereinigung (KBV) und der Arbeitsgemeinschaft der Wissenschaftlichen Medizinischen Fachgesellschaften (AWMF) seit 2003 Nationale Versorgungs-Leitlinien (NVL-Programm) erstellt $[4,28]$. Mittlerweile liegt für die Erstellung von VersorgungsLeitlinien der 4. Methodenreport vor [31]. In diesem Heft werden beispielhaft ein Update der interdisziplinären S2-Therapieleitlinie der Juvenilen Idiopathischen Arthritis (2. Auflage) [S. 386391 in dieser Ausgabe) und erstmals die Interdisziplinäre AWMF-Leitlinie zur Diagnostik von primären Immundefekten (S2k) [S. 378-385] wiedergegeben.

Die 12. Novelle des Arzneimittelgesetzes, die schrittweise Einführung des allgemeinverbindlichen Systems von Fallpauschalen zur Endtgeltung stationärer Behandlungen und die Einsetzung des Gemeinsamen Bundesausschusses (G-BA) als das oberste Beschlussgremium der gemeinsamen Selbstverwaltung der Ärzte, Zahnärzte, Psychotherapeuten, Krankenhäuser und Krankenkassen haben im zurückliegenden Jahrzehnt die deutsche Medizin besonders nachhaltig verändert. Aus der Sicht der pädiatrischen Onkologie und Hämatologie ist hierzu in der Klinischen Pädiatrie wiederholt berichtet worden $[1,6,15]$.

Speziell als Maßnahme zur Verbesserung und Sicherung der Struktur-, Prozess- und Ergebnisqualität hat der G-BA mit Wirkung zum 01.01.2007 für die Versorgung von Patienten im Alter von 0 bis $<18$ Jahren mit einer hämatoonkologischen Krankheit eine Vereinbarung beschlossen. Hierzu werden nicht nur die pädiatrischen Zentren für die hämatologisch-onkologische Versorgung aus fachlicher Sicht definiert, sondern gleichzeitig auch die strukturellen Mindestanforderungen deklariert [41].

Diese Gesetze und Vereinbarung haben sich auf die Zahl der hämato-onkologischen Behandlungszentren ausgewirkt, die Patienten an das Kinderkrebsregister in Mainz melden [10]. In den Jahren 1998-2000 waren dies etwa 100 Klinken, von denen ca. 50 Kliniken nur 1-9 Patienten gemeldet hatten [8]. In 2010 haben nur noch 65 pädiatrische Kliniken Meldungen an das Kinderkrebsregister in Mainz geschickt, und die Zahl der Kliniken mit $<10$ neu an Krebs erkrankten Kindern und Jugendlichen hat sich auf 12 verringert. Für diese Zentralisierung mögen unterschiedliche Faktoren eine Rolle gespielt haben, aber wahrscheinlich hat die Deklarierung von Mindestanforderungen an Struktur und Ausstattung der Kliniken dazu beigetragen.

\section{Krebskranke Jugendliche als Hochrisikogruppe \\ $\nabla$}

Da der G-BA-Beschluss die 15- bis < 18-jährigen Patienten mit Krebserkrankung explizit einbezieht, kommt den Jugendlichen und Heranwachsenden aus klinisch-wissenschaftlicher Sicht besondere Bedeutung zu. Dies ergibt sich auch aus den Daten des Krebsregisters des nationalen Krebsinstituts der USA (SEER - Surveillance, Epidemiology, and End Results). Da bei den Adoleszenten und den jungen Erwachsenen (16-39 Jahre) mit Krebs der Anstieg der Überlebenswahrscheinlichkeit hinter der von jüngeren bzw. älteren Patienten signifikant zurückbleibt, stellen diese eine Hochrisikogruppe dar [2]. Wood und Lee [42] belegen diese allgemeine Feststellung speziell für die hämatologischen Neoplasien und machen aufgrund ihrer umfangreichen Literaturübersicht mehrere Gründe dafür verantwortlich:

- eine größere Zahl therapiebedingter Komplikationen im Vergleich zu Kindern,

- der geringere Zugang zu einer auf das Alter abgestimmten klinischen Versorgung und hierbei insbesondere fehlende an die Bedürfnisse dieser Altersgruppe angepasste klinische Studien,

- eine niedrigere Compliance zu verordneten Medikamenten und Behandlungsplänen sowie

- die für diese Altersgruppe spezielle psychische Situation.

Deshalb bedürfen Jugendliche mit Krebserkrankung in der GPOH einer besonderen Beachtung $[7,8,23]$. Aufgabe der GPOH wird es sein, die wissenschaftlichen Fragestellungen noch stärker auf die verschiedenen Altersgruppen auszurichten.

\section{Erkenntnisgewinn bei Diagnosen mit geringen Fallzahlen \\ $\nabla$}

Die Pädiatrie ist wesentlich gekennzeichnet durch die große Zahl unterschiedlicher Diagnosen, die der Erwachsenenmedizin nicht nachsteht, und die in der Regel deutlich niedrigere Fallzahl pro Einzeldiagnose. Zwangsläufig haben 
kasuistische Mitteilungen, Analysen von kleinen Fallzahlen und retrospektive Auswertungen eine größere Bedeutung als in der Erwachsenenmedizin $[15,23]$. In Anbetracht der hohen Heilungsraten, wie sie durch die Kohorte der Langzeitüberlebenden des Kinderkrebsregisters belegt sind [9], werden prospektivrandomisierte Studien nur noch im internationalen Verbund möglich sein.

Das Hereditäre Hyperferritinämie-Katarakt-Syndrom [S. 346351] und die bösartigen Pankreastumoren [S. 341-345] sind bei Kindern und Jugendlichen sehr selten; beispielhaft kommt dem altersabhängigen Aspekt eine ganz unterschiedliche Bedeutung für die Diagnosestellung und die Prognoseeinschätzung zu.

\section{Hereditäres Hyperferritinämie-Katarakt-Syndrom}

Cario und Mitarb. stellen 5 Kinder aus Familien mit Hereditärem Hyperferritinämie-Katarakt-Syndrom [S. 346-351] vor, in denen jeweils mehrere Erwachsene ein- oder beidseitige Katarakte als einzige klinische Krankheitssymptome haben. Die ausführliche Darstellung der Patienten und ihrer Familien belegt, dass bei dieser dominant vererbten Krankheit die Manifestation und die Schwere des Kataraktes zwischen und innerhalb der Familien stark variieren können, obwohl bei den jeweiligen Familienmitgliedern immer dieselbe Punktmutation vorliegt. Weiterhin führten bei den Indexpatienten nicht Sehstörungen, sondern unklare abdominale Symptome bzw. eine Anämie zum Arztbesuch, sodass die Hyperferritinämie jeweils eher zufällig festgestellt wurde und dieser Schlüsselbefund trotz der positiven Familienanamnese nicht zur raschen Diagnose geführt hat. Bei einer sorgfältig erhobenen Familienanamnese hätten sich aufwendige Untersuchungen vermeiden lassen.

Ein anderer Aspekt ist der, dass im Kindesalter eine Hyperferritinämie bei unterschiedlichen gut- und bösartigen Erkrankungen vorkommt, die meist umso ungünstiger verlaufen, je jünger die Kinder sind $[20,30]$. Doch selbst bei akuter Lebensbedrohung sind zeitraubende diagnostische Umwege bei älteren Kindern nicht auszuschließen, wie ein ausführlicher Fallbericht eindringlich belegt [22]. Da bei rascher zielgerichteter Therapie sehr gute Heilungsaussichten bestehen, ist zusätzlich ein diagnostischer Algorithmus zu verwenden, der bei kranken Kindern auf routinemäßig zu erhebenden Laboruntersuchungen aufbaut [20]. Dieser hat das Hereditäre Hyperferritinämie-Katarakt-Syndrom zu berücksichtigen, obwohl dieses im Allgemeinen erst bei Erwachsenen Krankheitswert erlangt.

\section{Pankreastumoren}

Bösartige Pankreastumoren sind bei Erwachsenen häufig und haben im metastatischen Zustand meist eine ungünstige Prognose; deshalb sind sie Gegenstand intensiver klinischer Forschungen. Wegen ihrer Seltenheit bei Kindern und Jugendlichen fehlen prospektive Studien, sodass oft die Daten von Erwachsenen zur Grundlage therapeutischer Überlegungen und prognostischer Aussagen herangezogen werden. Brecht und Mitarb. [S. 341-345] haben die Datenbank von SEER für die von 1973-2004 gemeldeten Patienten im Alter von 0 bis $<30$ Jahren in 6 Altersgruppen (0-4, 5-9, 10-14, 15-19, 20-24, 25-29 Jahre) ausgewertet, um bei einer Mindestnachbeobachtungszeit von 5 Jahren validere Aussagen machen zu können. Trotz der langen Rekrutierungszeit enthält dieses große Register nur 228 junge Patienten mit malignen Pankreastumoren, die überwiegend den Gruppen der Heranwachsenden und jungen Erwachsenen angehören. Die Zahl der histologischen Subentitäten variiert erheblich in den Altersgruppen, und die Metastasierungsrate nimmt mit dem Alter signifikant zu. Das 5-Jahres-Überleben nach Tumorstadium beträgt $87 \%$ bei lokalisierten Tumoren $(n=54)$, $68 \%$ bei regionaler Metastasierung $(n=42)$ und $21 \%$ bei Fernmetastasen $(n=108)$. Die multivariate Analyse ergibt, dass Histologie, Tumorstadium und Alter unabhängige prognostische Faktoren sind.

Dies zeigt, dass die Daten von Pankreastumoren, die im Erwachsenenalter häufig sind, nicht im Verhältnis von 1:1 auf Kinder und Jugendliche zu übertragen sind. Dementsprechend kommen die Autoren zu dem Schluss, dass für weiterführende klinische Studien bei Kindern und Jugendlichen internationale Kooperationen notwendig sind. Hierfür stellt das internetbasierte Netzwerk für seltene Tumoren eine geeignete Plattform dar $[3,37]$.

\section{Welche Therapie ist für Jugendliche richtig? $\nabla$}

Konkret stellen sich die Fragen, ob bei einem 16-jährigen Jugendlichen Therapieentscheidungen anders ausfallen als bei einem 6-jährigen Kind oder 26-jährigen Erwachsenen und welche Gründe hierfür maßgeblich sind. Zur Beantwortung, werden beispielhaft biologische und therapeutische Erkenntnisse unter besonderer Berücksichtigung der 2. Lebensdekade bei der Akuten Lymphatischen Leukämie (ALL), dem Hodgkin-Lymphom, den Keimzelltumoren und der Fanconi-Anämie referiert.

\section{Akute Lymphatische Leukämie (ALL)}

Die ALL ist die häufigste maligne Diagnose bei Kindern, aber selten bei Erwachsenen. Diese früher infauste Erkrankung kann heute bei Kindern im Alter von 1-10 Jahren in etwa 90\% geheilt werden; bei jüngeren und älteren Patienten dagegen ist die Prognose deutlich schlechter. Diese seit Langem bekannte Feststellung, erfährt durch vergleichende Untersuchungen bei 5181 Kindern und Jugendlichen mit ALL im Alter von $0-<18$ Jahren, die zwischen 10/1986 und 06/1999 in die Therapieoptimierungsstudien ALL-BFM 86, 90 und 95 von über 80 teilnehmenden Zentren aufgenommen wurden, zumindest teilweise eine Erklärung [27]. Die Analyse der prognostischen Kriterien dieser prospektiv erfassten Patienten belegen, „dass sich die altersabhängig unterschiedliche Prognose der ALL-Gesamtgruppe zumindest teilweise auf die zwischen den Altersgruppen unterschiedliche Verteilung von prognostisch relevanten biologischen Subgruppen zurückführen lässt".. Diese Erkenntnis relativiert bei der ALL das Alter als unabhängigen Risikofaktor und belegt die Wichtigkeit der einheitlichen und standardisierten Diagnostik zytogenetischer, immunphänotypischer und molekularbiologischer Risikofaktoren durch ausgewiesene Referenzlabore, die innerhalb der GPOH hierfür und für andere Therapieoptimierungsstudien bestehen $[7,8,23]$.

\section{Hodgkin-Lymphom}

Beim Hodgkin-Lymphom, das im 1. Lebensjahrzehnt nur selten auftritt, haben nach einer Metaanalyse mit 85 Kohorten von Erwachsenen und 38 Kohorten von Kindern die pädiatrischen Patienten ein etwa $9 \%$ höheres ereignisfreies Überleben und Gesamtüberleben [14]. Dagegen können nach den Daten der deutschen Studiengruppe Adoleszenten (15-20 Jahre) mit vergleichbarem Erfolg wie junge Erwachsene (21-45 Jahre) mit den Protokollen für Erwachsene behandelt werden. Allerdings wurde ein geringfügig, aber statistisch signifikant höheres Risiko für maligne Sekundärerkrankungen bei jungen Erwachsenen gegenüber Adoleszenten beobachtet [11]. Die Reduktion von 
malignen Sekundärerkrankungen, kardialen Spätfolgen und Infertilität sind Studienziele der pädiatrischen DAL-HD-Protokolle seit $1978[33,35,36]$. Konsekutiv sind deshalb in randomisierten Studien die Strahlendosen und die Strahlenfelder reduziert sowie die Kombination der Zytostatika modifiziert worden [26,34]. In diesem Heft beschreiben Kluge und Körholz wie mittels des FDG-PET beim pädiatrischen Hodgkin-Lymphom [S. 315-319] die Bestrahlungen abhängig vom Therapieansprechen weiter reduziert werden sollen und so eine den individuellen Erfordernissen genau angepasste Therapie verabreicht wird.

\section{Keimzelltumoren}

Völlig anders ist die Situation bei Keimzelltumoren, bei denen 5 verschiedene Subentitäten (Teratome, Seminome/Dysgerminome, embryonale Karzinome, Dottersacktumoren und Choriokarzinome) mit unterschiedlicher Dignität und unterschiedlicher Sensibilität gegenüber Strahlen- und Chemotherapie vorkommen. Sie sind bei jungen Männern (15-45 Jahre) der kaukasischen Bevölkerungsgruppe die häufigste bösartige Tumorgruppe überhaupt und manifestieren sich bei diesen ganz überwiegend in den Hoden. Im metastasierten Stadium handelte es sich bei den nichtseminomatösen Keimzelltumoren um eine infauste Erkrankung, bis mit der Kombination von Vinblastin, Bleomycin und Cisplatin durch Einhorn und Donohue 1977 der therapeutische Durchbruch gelang [12]. Bei Kindern und Jugendlichen $<15$ Jahre dagegen gehören die Keimzelltumoren mit 3-4\% aller Neuerkrankungen zu den seltenen Tumoren [10] und sind nur zu 20\% in den Hoden lokalisiert [38]. Therapeutisch reichen bei Kindern meist niedrigere Medikamentendosen - ausgenommen Platinderivate - zur Heilung metastasierter oder ungünstig lokalisierter Keimzelltumoren aus, als sie bei Erwachsenen erforderlich sind [17].

Da das Vorkommen der verschiedenen histologischen Subentitäten und die Tumorlokalisationen in charakteristischer Weise in Abhängigkeit vom Alter variieren, wurden vergleichende zytogenetische und molekulargenetische Untersuchungen erstellt, die gravierende Unterschiede erbrachten $[39,40]$. Dies überrascht, da die o.g. Subentitäten bei Kindern, Jugendlichen und Erwachsenen mikroskopisch jeweils gleich aussehen [29].

Diese zytogenetischen Unterschiede sind geeignet, das unterschiedliche biologische Verhalten der Keimzelltumoren bei Kindern, Jugendlichen und Erwachsenen zu erklären [29]. So war in der Pediatric Intergroup Study das Überleben der Jugendlichen (12-18,5 Jahre) mit malignen extragonadalen Keimzelltumoren in der univariaten Analyse signifikant schlechter als bei den jüngeren Patienten (5-Jahre-OS, $53,7 \% \pm 14,9 \%$ vs. $88,5 \% \pm 3,4 \%$; $\mathrm{P}<0,0001$ ) [25]. Da diese Tumoren direkt von den primordialen Stammzellen abstammen sollen [24], sind sie zusätzlich von besonderem Interesse für die Erforschung der Onkogenese.

\section{Fanconi-Anämie}

Die sehr seltene Fanconi-Anämie ist eine $\mathrm{x}$-chromosomal oder autosomal rezessiv vererbbare DNA-Reparaturerkrankung, bei der es zu einem charakteristischen Krankheitsbild mit angeborenen Fehlbildungen, einem progredienten Versagen des hämatopoetischen Systems und einer stark gehäuften Entwicklung von Neoplasien in verschiedenen Organsystemen kommt. Mittlerweile sind durch die Komplementationsdiagnostik 15 verschiedene Keimbahnmutationen (FANCA/B/C/D1/D2/E/F/G/I/J/L/ $\mathrm{M} / \mathrm{N} / \mathrm{O} / \mathrm{P}$ ) identifiziert worden [21].

Diagnostisch wichtig ist, dass sich bei einer doppelt heterozygoten Fanconi-Anämie die Manifestation der Erkrankung in das
Erwachsenenalter verschieben kann, da die typischen klinischen Stigmata fehlen können oder so diskret sind, dass sie nicht zur frühen Diagnose führen. Erst aufgrund der hämatologischen Veränderungen wird dann im fortgeschrittenen Alter die Diagnose gestellt. Beispielhaft ist eine Patientin, bei der die FanconiAnämie nach 2 onkologischen Erkrankungen mit entsprechenden zytostatischen Behandlungen im 6. Lebensjahrzehnt durch die Kooperation von internistischen und pädiatrischen HämatoOnkologen gestellt wurde [18]. Therapeutisch kurativ ist die hämatopoetische Stammzelltransplantation, die wegen der exzessiv erhöhten Chromosomenbrüchigkeit, der langen Krankheitsdauer und der progredienten Infektionsanfälligkeit riskant ist. Die konditionierende Therapie vor der Stammzelltransplantation und die Art des Transplantates selbst sind entscheidend für den Erfolg [19]. Wie sehr die Konditionierung bei unterschiedlichen Immundefekten bei welcher Art von Transplantat variieren kann, wird aus der Übersicht von Hönig et al. [S. 320-325] ersichtlich.

Für die Grundlagenforschung ist die Fanconi-Anämie von besonderem Interesse, da bei Vorhandensein eines Fanconi-AnämieGens das Krebsrisiko vielfach erhöht ist. Beispielsweise ist die FANCD1-Keimbahnmutation mit dem Brustkrebsgen BRCA2 identisch, das zum familiärem Brust- und Eierstockkrebs führt, während das kürzlich entdeckte FANCN- oder auch PALB2-Gen zu Krebs bei Kindern prädisponiert ist [32].

\section{Ausblick}

$\nabla$

Die Beispiele zeigen, dass jede Erkrankung ihre eigene Biologie hat, die wesentlich durch das Alter modifiziert wird. Dies kann - wie bei der ALL - die Verteilung der unterschiedlichen Prognosegruppen betreffen oder bei den Keimzelltumoren genetisch differente Tumoren bedeuten. Unabhängig von den therapeutischen Implikationen, die für den Einzelnen entscheidend sind, kommt dem Erkenntnisgewinn für die Gesamtheit der Patienten Bedeutung zu. Dies setzt allerdings voraus, dass die Bedingungen möglichst gut vergleichbar sind, damit keine Verzerrungen auftreten. Dies wird besonders beim Hodgkin-Lymphom deutlich, wo der Vergleich zwischen behandelten Kindern und Adoleszenten, die nach pädiatrischen Protokollen behandelt sind, unter den geringeren Fallzahlen leidet [14]. Insofern ist es nicht unerheblich, dass seit 2007 nach dem G-BA-Beschluss Adoleszenten ( 15 bis < 18 Jahre) in pädiatrisch-hämato-onkologischen Zentren zu behandeln sind. Hierdurch ist zu erwarten, dass die bei 10-18 Jahre alten Patienten zur Beantwortung anstehenden Fragen schneller und mit der nötigen Power beantwortet werden können, da die erforderlichen Fallzahlen rascher erreicht werden.

Die SEER-Daten aus den USA lassen sich wegen der unterschiedlichen Rahmenbedingungen nur bedingt auf die deutschen Verhältnisse übertragen. Gerade deshalb bedürfen die in den USA beobachteten Unterschiede in der Überlebenswahrscheinlichkeit von Kindern, Jugendlichen und jungen Erwachsenen aber dringend einer Klärung. Diese wird in Deutschland für die Adoleszenten und jungen Erwachsenen wesentlich von den Meldungen an das Kinderkrebsregister einerseits und an die verschiedenen epidemiologischen Krebsregister der einzelnen Bundesländer für Erwachsene andererseits abhängen. Das Kinderkrebsregister erfasst in Deutschland die krebskranken Kinder und Jugendliche flächendeckend, die $\mathrm{zu}>90 \%$ nach den kooperativen, multizentrischen, prospektiven Therapieoptimierungspro- 
tokollen und -studien der GPOH behandelt werden [7,8,10]. Da die Überlebensdaten der Kohorte der Langzeitüberlebenden für konsekutive Behandlungsperioden und verschiedene Altersgruppen (0-14 Jahre) schon verfügbar sind [9], darf damit gerechnet werden, dass in absehbarer Zeit auch entsprechende Daten für die 15- bis 18-jährigen Patienten zur Verfügung stehen werden.

Interessenkonflikt: Die Autoren erklären hiermit, dass kein Interessenkonflikt besteht.

\section{Literatur}

1 Berthold F, Bode G, Böcker A et al. Maßnahmen zur Qualitätssicherung für die stationäre kinderonkologische Versorgung. Klin Padiatr 2006; 218: 293-295

2 Bleyer A. Young adult oncology: the patients and their survival challenges. CA Cancer J Clin 2007; 57: 242-255

3 Brecht IB, Graf N, Schweinitz von D et al. Networking for children and adolescents with very rare tumors: Foundation of the GPOH Pediatric Rare Tumor Group. Klin Padiatr 2009; 221: 181-185

4 Bundesärztekammer (BÄK), Kassenärztliche Bundesvereinigung (KBV), Arbeitsgemeinschaft der Wissenschaftlichen Medizinischen Fachgesellschaften (AWMF). Vertrag über eine Kooperation zum Zwecke der Entwicklung und Beschlussfassung im Rahmen des Nationalen Programms für Versorgungs-Leitlinien. Köln: 2003 [cited: 2010 Mai 07] http://www.aezq.de/edocs/pdf/vertraege/awmfvertragfinal.pdf

5 Calaminus G, Schneider DT, Bökkerink JP et al. Prognostic value of tumor size, metastases, extension into bone, and increased tumor marker in children with malignant sacrococcygeal germ cell tumors: a prospective evaluation of 71 patients treated in the German cooperative protocols Maligne Keimzelltumoren (MAKEI) 83/86 and MAKEI 89. J Clin Oncol 2003; 21: 781-786

6 Christaras A, Schaper J, Strelow $H$ et al. Auswirkungen des selbstlernenden G-DRG-Systems 2004-2006 auf die Vergütung stationärer Leistungen bei pädiatrisch-hämatologisch-onkologischen Patienten am Beispiel einer Universitätsklinik. Klin Padiatr 2006; 218: 366-378

7 Creutzig $U$, Herold $R$, Henze G. Ergebnisse des Kompetenznetzes Pädiatrische Onkologie und Hämatologie - ein Rückblick. Klin Padiatr 2010; 222: 333-336

8 Creutzig $U$, Jürgens $H$, Herold $R$ et al. Konzepte der GPOH und des Kompetenznetzes zur Weiterentwicklung und Qualitätssicherung in der Pädiatrischen Onkologie. Klin Padiatr 2004; 216: 379-383

9 Debling D, Spix C, Blettner $M$ et al. The cohort of long-term survivors at the German Childhood Cancer Registry. Klin Padiatr 2008; 220: 371-377

10 Deutsches Kinderkrebsregister. http://www.kinderkrebsregister.de/

11 Eichenauer DA, Bredenfeld H, Haverkamp $H$ et al. Hodgkin's lymphoma in adolescents treated with adult protocols: A report from the German Hodgkin study group. J Clin Oncol 2009; 27: 6079-6085

12 Einhorn LH, Donohue JP. Improved chemotherapy in disseminated testicular cancer. U Urol 1977; 117: 65-71

13 Europarat, Verbindung der Schweizer Ärztinnen und Ärzte, Ärztliche ZentralstelleQualitätssicherung, Ludwig Boltzmann Institut für Krankenhausorganisation. Entwicklung einer Methodik für die Ausarbeitung von Leitlinien für optimale medizinische Praxis. Empfehlung Rec (2001)13 des Europarates am 10. Oktober 2001 und Erläuterndes Memorandum. Deutschsprachige Ausgabe. Z Arztl Fortbild Qualitatssich 2002; 96 ( suppl iii): 3-60

14 Franklin J. Do children with Hodgkin's disease have a better prognosis than adults? Application of a generalised linear model to a systematic review of published results. In: Hasman A, Blobel B, Dudeck J. et al. (eds.). Medical Infobahn for Europe - Proceedings of MIE 2000 and GMDS 2000. Amsterdam: IOS Press, 2000

15 Graf N, Göbel U. Therapieoptimierungsstudien der Gesellschaft für Pädiatrische Onkologie und Hämatologie (GPOH) und 12. Novelle des Arzneimittelgesetzes zur Umsetzung der EU-Richtlinie. Klin Padiatr 2004; 216: 129-131

16 Göbel $U$, Gortner L. Klinische Forschung und Behandlung in der Pädiatrie. Klin Padiatr 2008; 220: 2-4

17 Göbel U, Schneider DT, Calaminus $G$ et al. Germ cell tumors in childhood and adolescence. GPOH MAKEI and the MAHO study groups. Ann Oncol 2000; 11: 263-271

18 Huck K, Hanenberg H, Gudowius S et al. Delayed diagnosis and complications of Fanconi anaemia at advanced age - a paradigm. $\mathrm{Br}$ J Haematol 2006; 133: 188-197
19 Huck K, Hanenberg H, Nürnberger $W$ et al. Favourable long-term outcome after matched sibling transplantation for Fanconi-anemia (FA) and in vivo T-cell depletion. Klin Padiatr 2008; 220: 147-152

20 Janka $G$. Hemophagocytic lymphohistiocytosis: When the immune system runs amok. Klin Padiatr 2009; 221: 278-285

21 Kitao H, Takata M. Fanconi anemia: a disorder defective in the DNA damage response. Int J Hematol 2011; 93: 417-424

22 Kerzel S, Zemlin M, Kömhoff $M$ et al. Hyperferritinemia as the diagnostic clue in life-threatening hemophagocytic lymphohistiocytosis. Klin Padiatr 2009; 221: 318-321

23 Klingebiel T, Creutzig U, Christaras et al. Meilensteine der Gesundheitspolitik und seltene Erkrankungen. Klin Padiatr 2010; 222: 121-123

24 Looijenga LHJ. Embryogenesis and metastatic testicular germ cell tumors of adolescents. Klin Padiatr 2009; 221: 134-135

25 Marina $N$, London WB, Lindsay Frazier A et al. Prognostic factors in children with extragonadal malignant germ cell tumors: A pediatric intergroup study. J Clin Oncol 2006; 24: 2544-2548

26 Mauz-Körholz C, Hasenclever D, Dörffel W et al. Procarbazine-free OEPA-COPDAC chemotherapy in boys and standard OPPA-COPP in girls have comparable effectiveness in pediatric Hodgkin's lymphoma: the GPOH-HD-2002 study. J Clin Oncol 2010; 28: 3680-3686

27 Möricke A, Zimmermann M, Reiter A et al. Prognostic impact of age in children and adolescents with acute lymphoblastic leukemia: Data from the trials ALL-BFM 86, 90, and 95. Klin Padiatr 2005; 217: $310-320$

28 Ollenschläger G, Berenbeck C, Löw A et al. Nationales Programm für Versorgungs-Leitlinien bei der Bundesärztekammer - MethodenReport. Z Ärztl Fortbild Qualitätssich 2002; 96: 545-548

29 Oosterhuis J, Looijenga $L$. Testicular germ-cell tumours in a broader perspective. Nat Rev Cancer 2005; 5: 210-222

30 Pachlopnik Schmid J, Basile G de Saint. Inherited hemophagocytic lymphohistiocytosis (HLH). Klin Padiatr 2010; 222: 345-350

31 Programm für Nationale VersorgungsLeitlinien. http://www.versor gungsleitlinien.de/methodik/pdf/nvl_methode_4.aufl.pdf der 4. Methodenreport

32 Reid S, Schindler D, Hanenberg $\mathrm{H}$ et al. Biallelic mutations in PALB2 cause Fanconi anemia subtype FA-N and predispose to childhood cancer. Nat Genet 2007; 39: 162-164

33 Rendtorff R, Hohmann C, Reinmuth $S$ et al. Hormone and sperm analyses after chemo- and radiotherapy in childhood and adolescence. Klin Padiatr 2010; 222: 145-149

34 Schellong G, Pötter R, Brämswig J et al. High cure rates and reduced long-term toxicity in pediatric Hodgkin's disease: the German-Austrian multicenter trial DAL-HD-90. The German-Austrian Pediatric Hodgkin's Disease Study Group. J Clin Oncol 1999; 17: 3736-3744

35 Schellong $G$, Riepenhausen $M$, Bruch $C$ et al. Late valvular and other cardiac diseases after different doses of mediastinal radiotherapy for Hodgkin Disease in children and adolescents: Report from the longitudinal GPOH follow-up project of the German-Austrian DAL-HD studies. Pediatr Blood Cancer 2010; 55: 1145-1152

36 Schellong G, Waubke-Landwehr AK, Langermann HJ. Prediction of splenic involvement in children with Hodgkin's disease. Significance of clinical and intraoperative findings. A retrospective statistical analysis of 154 patients in the German therapy study DAL-HD-78. Cancer 1986; 57: 2049-2056

37 Schneider DT, Brecht IB. Care for rare cancers: Improved care requires improved communication. Klin Padiatr 2010; 222: 124-126

38 Schneider DT, Calaminus G, Koch $S$ et al. Epidemiological analysis of 1442 children and adolescents registered in the German Germ Cell Tumor Protocols. Pediatr Blood \& Cancer 2004; 42: 169-175

39 Schneider DT, Schuster AE, Fritsch MK et al. Comparative genomic hybridization of childhood germ cell tumors. Klin Padiatr 2001; 213: 204-211

40 Schneider DT, Zahn S, Sievers S et al. Molecular genetic analysis of central nervous system germ cell tumors with comparative genomic hybridization. Modern Pathology 2006; 19: 864-873

41 Vereinbarung des Gemeinsamen Bundesausschusses über Maßnahmen zur Qualitätssicherung für die stationäre Versorgung von Kindern und Jugendlichen mit hämato-onkologischen Krankheiten gemäß § 137 Abs. 1, Satz 3 Nr. 2 SGB V für nach § 108 SGB V zugelassenen Krankenhäuser [Vereinbarung zur Kinderonkologie] vom 16. Mai 2006, In-Kraft-Treten am 1. Januar 2007). http://www.g-ba.de/ informationen/beschluesse/290

42 Wood WA, Lee SJ. Malignant hematologic diseases in adolescents and young adults. Blood 2011; 117: 5803-5815 